\title{
A ATUAÇÃO DA FISIOTERAPIA NA LUXAÇÃO TRAUMÁTICA DE JOELHO
}

\author{
Haron Silva Dorta*, Elizabeth Honorato Torato**, Adriana Lopes da Silva***, José Carlos de Araujo*** \\ Autor correspondente: Haron Silva Dorta - haron.dorta@unifesp.br \\ * Fisioterapeuta, Especialista e Mestrando em Ciências da Saúde pela Universidade Federal de São Paulo \\ ** Fisioterapeuta, Especialista pelo Hospital do Servidor Público Estadual - "Francisco Morato de Oliveira” \\ *** Fisioterapeuta graduado na Universidade do Grande ABC \\ O trabalho foi realizado no Departamento de Reabilitação em Ortopedia e Traumatologia Clínica da APRAESPI (Associação \\ de Prevenção, Atendimento Especializado e Inclusão da Pessoa com Deficiência de Ribeirão Pires)
}

\begin{abstract}
Resumo
A Luxação Traumática do Joelho é definida como a perda anatômica normal da articulação fêmur-tibial. Esse tipo de lesão está associado a uma força traumática elevada geralmente associada com lesões de alta velocidade, acidentes de trânsito, principalmente rodoviárias. O deslocamento também pode ocorrer a partir de lesões de baixa velocidade, como na pratica de esportes. O tratamento dessas lesões ainda é controverso e a atuação fisioterapêutica é inexistente na literatura. Esse estudo é um relato de caso onde a paciente L.A.V 56 anos, sexo feminino, sofreu um acidente automobilístico, onde foi atropelada por uma motocicleta. Com o diagnóstico de luxação traumática no joelho decorrente do acidente, a paciente realizou fisioterapia por 12 semanas. Conclusão: A paciente apresentou melhora em seu quadro clínico onde voltou a realizar suas atividades diárias sem dificuldades. Mostrando que o papel da fisioterapia foi fundamental neste tipo de caso. A reabilitação deste paciente minimizou também a necessidade da realização da cirurgia de reconstrução ligamentar. Outros estudos devem ser realizados, para complementar e confirmar nossos resultados.
\end{abstract}

Palavras-chave: Luxação do Joelho; Fisioterapia; Reabilitação.

\section{THE ROLE OF PHYSIOTHERAPY IN TRAUMATIC KNEEDISLOCATION}

\begin{abstract}
The Traumatic knee dislocation is defined as the normal anatomical loss of femoral-tibial joint. This type of injury is associated with a traumatic force high usually associated with high-speed injuries, traffic accidents, especially road. The displacement can also occur from low speed lesions, as in the practice
\end{abstract}


of sports. The treatment of these lesions is still controversial and the physiotherapy performance is lacking in the literature. This study is a case report where the patient $\operatorname{LAV}{ }_{56} 6$, female, suffered a car accident where he was hit by a motorcycle. With the diagnosis of traumatic dislocation knee resulting from the accident, the patient underwent physical therapy for 12 weeks. Conclusion: The patient showed improvement in their clinical condition where again perform daily activities without difficulty. Showing that the role of physical therapy was crucial in this type of case. The rehabilitation of this patient also minimized the need to perform ligament reconstruction surgery. Other studies are needed to complement and confirm our results

Keywords: Knee Injuries; Knee Dislocation, Physiotherapy; Rehabilitation.

\section{INTRODUÇÃO}

A Luxação Traumática do joelho (LT)) é uma lesão relativamente rara onde representa aproximadamente $0,02 \%$ de todas as lesões ortopédicas. ${ }^{(1,2)}$ Este tipo de lesão não hã consenso na literatura, o que faz ter o seu tratamento controverso. ${ }^{(3)}$

O papel da fisioterapia nas lesões ligamentares do joelho é bem estabelecido na literatura, bem como seus inúmeros benefícios no período pré e pós operatório. ${ }^{(4)}$ Já há luxação traumática do joeIho se tornam desconhecida e limitada no processo de reabilitação, devido a sua raridade, e inexistência de estudos na literatura sobre o assunto. $\mathrm{O}$ que faz deste artigo o primeiro a relatar tal caso, assim como os seus benefícios.

\section{A ARTICULAÇÃO DO JOELHO}

A articulação do joelho pode ser descrita como gínglimo ou articulação em dobradiça, (5) que é uma articulação de sobrecarga, que une dois ossos longos com superfícies articulares pouco congruentes, estando sujeito a inúmeras lesões traumáticas, sendo uma das articulações mais lesadas em nosso corpo. ${ }^{(5)} \mathrm{A}$ luxação traumática do joelho geralmente acarreta lesões do ligamento cruzado anterior (LCA), ligamento cruzado posterior (LCP) e pelo menos um complexo ligamentar colateral. ${ }^{(5)}$ Os ligamentos cruzados têm funções de assegurar a estabilidade articular no sentido anteroposterior, permitir os movimentos de flexo-extensão, mantendo as superfícies articulares do fêmur e da tíbia em contato e restringir os movimentos nos planos frontais e transversal.(5)

O joelho é uma articulação envolvida em inúmeras patologias e lesões. Suas estruturas anatômicas como meniscos, ligamentos e cápsula articular são fundamentais para o desenvolvimento de um movimento biomecânico sem nenhuma imperfeição, onde no ato de uma lesão de qualquer estrutura, a limitação funcional e a marcha são alteradas acometendo todo o aparelho locomotor. ${ }^{(5)}$

\section{LUXAÇÃO TRAUMÁTICA DO JOELHO}

A luxação traumática do joelho (LTJ) é definida como a perda da relação anatômica normal da articulação fêmoro-tibial. Sendo classificada como anterior, posterior, lateral, medial ou mista, de acordo com o desvio da tíbia em relação ao fêmur. Quanto à apresentação inicial, as luxações de joelho podem ser óbvias (com a articulação ainda luxada) ou ocultas (com a articulação já reduzida espontaneamente). Rockwood preconiza que as luxações óbvias devem ser reduzidas imediatamente, antes mesmo do exame radiográfico. ${ }^{(6)} \mathrm{A}$ LTJ é uma lesão incomum. A sua incidência pode ser maior do que o registrado por causa daredução espontânea ou devi- 
do à redução realizada no local do acidente. A maioria dos deslocamentos ocorre como um resultado de lesões de alta velocidade, acidentes de trânsito, principalmente rodoviárias, podendo ocorrer também a partir de lesões de baixa velocidade, como na prática dos esportes. ${ }^{(7)}$ Com o trauma ocasionado, alterações articulares podem ocorrer como a instabilidade articular causada pela lesão ligamentar. A associação com lesão arterial e nervosa é outro fator que pode agregar mais gravidade à luxação do joelho. Outra lesão que é de grande preocupação é a lesão do nervo fibular, proporcionando a perda da função da musculatura dos compartimentos anterior e lateral da perna, como também alterações sensitivas dos dermátomos correspondentes. A lesão da artéria poplítea necessita de um atendimento precoce, sob risco da isquemia do membro inferior e possibilidade de amputação. ${ }^{(8)}$

A forma de classificação corriqueiramente usada na clínica e na literatura é a de Kennedy que é definida de acordo com a posição anatômica do membro distal e está comparando a o segmento proximal acometido. (9) Já Schenck define sua classificação de acordo com o numero de estruturas acometidas durante a luxação. ${ }^{(10)}$ Porém a forma do tratamento da luxação traumática do joelho é um campo da traumatologia ortopédica repleto de dúvidas e controvérsias. $O$ estabelecimento de conduta padronizada de tratamento para as lesões causadas pela luxação vem sendo motivo de estudo nos centros especializados. Durante o atendimento de trauma- tismos ortopédicos complexos. A baixa prevalência de luxação traumática do joelho e a falta de heterogeneidade de suas lesões têm sido os principais obstáculos para a interpretação dos resultados. ${ }^{(6)}$

\section{RELATO DE CASO}

A paciente L.A.V 56 anos, sexo feminino, 1.54 metros, aposentada, sedentária, IMC 35.42, foi atropelada por uma motocicleta, onde veio a sofrer uma luxação traumática no joelho esquerdo. Foi socorrida e não foi feita a redução imediatamente, e sim levada para um hospital de referência em ortopedia e traumatologia. A paciente foi levada ao centro cirúrgico, onde foi avaliada, e apresentava uma fratura Schatzker(11) tipo I com desvio inferior a 3 milímetros e lesões no ligamento cruzado anterior LCA, ligamento colateral medial LCM, não houve lesão na artéria poplítea, nem no nervo fibular. Seguiu para o centro cirúrgico onde foi anestesiada, feita a redução do joelho e a estabilização com fixador ex-terno transarticular no membro inferior acometido por apresentar a fratura e uma instabilidade articular elevada, a fixação foi feita como forma de controle do dano ortopédico inicial. Como demonstrado na Figura 1 com o Raio-X (Rx) da paciente com luxação traumática do joelho e na Figura 2 o Rx do joelho com fixador externo trans-articular da paciente para estabilização articular.
Figura 1 - RX da luxação traumática do joelho

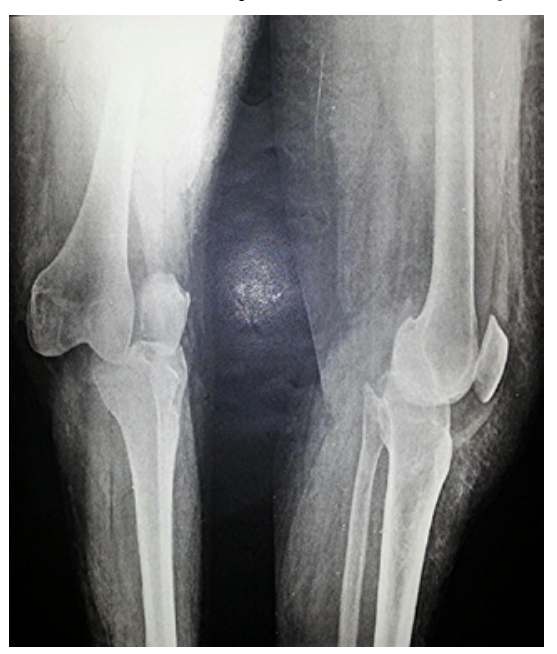

Figura 2 - Rx do fixador externo transarticular

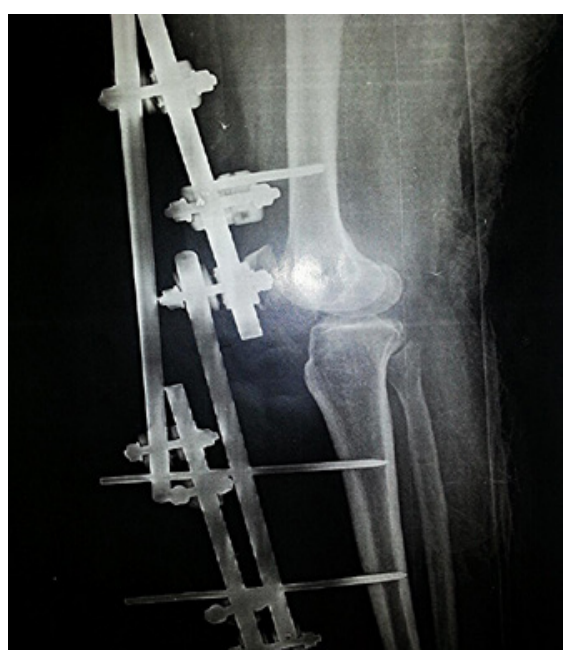


Após a cirurgia a paciente L.A.V ficou acamada por 02 meses com o fixador e não realizou nenhum tipo de tratamento, após a retirada deste fixador foi instruída a realizar exercícios em sua casa porém mesmo sem o fixador, estava somente em cadeira de roda. Depois de 02 semanas e conseguindo a deambular com o andador de tradicional de 04 apoios (standard) e deu início a fisioterapia. A sua avaliação foi realizada, no início e no termino do tratamento de fisioterapia.

A paciente apresentava na avaliação inicial, fraqueza muscular nos membros inferiores, e 21 pontos da escala de equilíbrio de Berg-EEB ${ }^{(12)}$ e Tegner Lysholm ${ }^{(13)}$ com score de 19, utilizava andador e com sua amplitude de movimento (ADM) em joeIho acometido com flexão de $39^{\circ}$ e extensão de $15^{\circ}$ para chegar em $0^{\circ}$.

\section{TRATAMENTO}

De acordo com a avaliação realizada no Departamento de Reabilitação em Ortopedia e Traumatologia Clínica da APRAESPI. A paciente realizou trabalho de ganho de força e resistência nos membros inferiores em cadeia cinética aberta (4 series $x 12$ repetições com 02 quilos), fortalecimento do tipo ativo-assistidos, ativo-livres e isométricos da musculatura de quadríceps, isquiotibiais, adutores, abdutores do quadril e glúteos (4x16 com 1 quilos), buscado o equilíbrio muscular. Eram rea- lizados também, mobilização patelar, séries de alongamento de flexores de quadril, quadríceps, isquiotibiais e glúteos ( $5 \times 10$ por 25 segundos) e treino de marcha em barra paralela com obstáculos. Os exercícios realizados do tipo proprioceptivos eram em dynadisc (5 minutos) e cama elástica (2xiominutos).

Gradativamente com a evolução do paciente, foi substituído o andador de 4 apoios por uma bengala de 1 apoio. A paciente também apresentou ganho de ADM em flexão e extensão de joelho dando inicio assim ao treino de marcha funcional com obstáculos, escadas e rampas. Os treinos proprioceptivos foram associados a movimentos rotacionais do paciente e de coordenação dos membros superior e treinos com os olhos fechados aumentando o grau de dificuldade, associando o treinamento proprioceptivo com treino para o equilíbrio. $\mathrm{Na}$ fase final do tratamento a paciente realizava os exercícios de maneira ativa assistida. Sem dificuldade em suas séries de treinamento, assim como apresentava uma marcha mais estável e com diminuição da oscilação do tronco látero lateral.

\section{RESULTADO}

Os resultados apresentado com a paciente durante o período de tratamento demonstrou sua melhora tanto em força muscular (Tabela 1), com ganho de ADM (Tabela 2), e equilíbrio (Tabela 3).

Tabela 1 - A força muscular

\begin{tabular}{ccc}
\hline Músculos avaliados & A & B \\
\hline Quadríceps & 4 & 5 \\
Isquiotibiais & 3 & 5 \\
Abdutor da coxa & 4 & 5 \\
Adutor da coxa & 3 & 5 \\
Glúteo Médio & $3+$ & 5 \\
Glúteo Maximo & $3+$ & 5 \\
\hline
\end{tabular}

A. Início do tratamento B. Termino do Tratamento 
Tabela 2 - Amplitude de movimento

\begin{tabular}{ccc}
\hline MOVIMENTo ARTICULAR & A & B \\
\hline Flexão do joelho & $39^{\circ}$ & $108^{\circ}$ \\
Extensão do joelho & $-15^{\circ}$ & $-4^{\circ}$ \\
\hline
\end{tabular}

A. Início do tratamento B. Término do tratamento

Tabela 3 - Avaliações por protocolos específicos

\begin{tabular}{ccc}
\hline Questionário & A & B \\
\hline Berg-EEB & 21 & 42 \\
Tegner Lysholm & 33 & 88 \\
\hline
\end{tabular}

A. Início do tratamento B. Término do tratamento

Os resultados encontrados mostraram os benefícios da fisioterapia em relação à LTJ, após a avaliação clínica foi decidida a não realização da cirurgia para a reconstrução dos ligamentos do joelho.

\section{DISCUSSÃO}

$\mathrm{Na}$ reabilitação a inexistência de estudo sobre o tema é um agravante em relação a pesquisas, o que faz deste trabalho o pioneiro a demonstrar os benefícios da fisioterapia na LTJ. Os resultados encontrados mostraram melhora em seu quadro de força muscular em membros inferiores, melhora no equilíbrio comprovado através da escala de Berg-EEB ${ }^{(12)}$ e melhora com o questionário de propriedades de medida especifico para sintomas do joelho. ${ }^{(13)}$

O tratamento dessas lesões ainda é controverso e há vários protocolos descritos. Porém, o tratamento cirúrgico seguido da reabilitação fisioterapêutica tem demonstrado melhores resultados quando comparado ao tratamento conservador de lesões ligamentares. ${ }^{(7,8)} \mathrm{O}$ procedimento de operar todos os grupos ligamentares lesados, no primeiro momento ou aguardar para um segundo momento, é discutido por vários autores. . $^{(6,1-16)}$ Aqueles que seguem a linha de um único procedimento cirúrgico defendem que, desta forma, o joelho adquire mais estabilidade. No entanto, aqueles que recomendam a cirurgia seriada, argumentam que os resultados em termos de ADM são melhores e apresentam menos artrofibrose. Apesar das divergências, há o consenso de que o tratamento cirúrgico oferece resultados funcionais mais eficazes, em relação ao conservador $^{(6,14,17)}$ no que diz respeito à ADM, estabilidade e adaptação do paciente às atividades cotidianas. ${ }^{(6,14,17)}$

Já Richter et al.(17) afirmam que, um programa de reabilitação bem conduzido tem valor prognóstico fundamental e indispensável ao paciente. ${ }^{(6,17)} \mathrm{Em}$ um estudo clínico onde comparava a reabilitação com reconstrução precoce do LCA versus reabilitação com reconstrução tardia do LCA, demonstrou que não houve diferença significativa entre os grupos. Porém, dos 62 indivíduos submetidos à reconstrução precoce, um não precisou realizar a cirurgia, enquanto que dos 59 da reconstrução tardia, 36 não precisaram realizá-la. ${ }^{18,19)}$ Isso demonstra que a fisioterapia pode diminuir a necessidade de intervenção cirúrgica em pacientes com lesão do LCA. ${ }^{199}$ Esses achados vãos de acordo com a literatura que mostra que a fisioterapia apresenta um papel fundamental para que o indivíduo recupere a funcionalidade, através da utilização de técnicas como cinesioterapia, eletroterapia, crioterapia e outras técnicas apropriadas para este tipo de lesão. ${ }^{(20)}$. 


\section{CONCLUSÃO}

Os resultados encontrados demonstraram que a atuação fisioterapêutica foi benéfica e com excelentes resultados na paciente com LTJ. Os benefícios na pacientes foram tão significativos, onde após o tratamento de 12 semanas, foi decidido a não realização cirúrgica para reconstrução ligamentar. Desta maneira demonstramos que a atuação da fisioterapia no paciente com LTJ é de grande importância e que seu papel na reabilitação de pacientes com lesão ligamentar no joelho no período pré operatório é satisfatório e em alguns casos pode evitar a necessidade de intervenção cirúrgica.

\section{AGRADECIMENTOS}

Queremos agradecer a APRAESPI pelo apoio na elaboração deste estudo, assim como as fisioterapeutas Carla Andreoli Dias, Iara Lima de Almeida e Michelle Niwa de Oliveira por sua contribuição, apoio e tempo dedicado a esse estudo.

\section{REFERÊNCIAS}

1. Rihn JA et al. The acutely dislocated knee: evaluation and management. J Am Acad Orthop Surg. 2004; 12: 334-346.

2. Kohl S, Stock A, e cols Dynamic intraligamentary stabilization and primary repair: A new concept for the treatment of knee dislocation. Injury. 2014. Oct 16. pii: SOO2O1383(14)00496-3.

3. Harner CD, Waltrip RL, Bennett $\mathrm{CH}$, Francis KA, Cole B, Irrgang JJ. Surgical management of knee dislocations. J Bone Joint Surg Am. 2004; 86-A (2):262-73.

4. Logerstedt DS, e cols Snyder-Mackler L. Orthopedic Section of the American Physical Therapy Association. Knee pain and mobility impairments: meniscal and articular cartilage lesions. J Orthop Sports PhysTher. 2010;4O(6):A1-A35.
5. Dorta HS. A atuação da hidroterapia na lesão do ligamento cruzado. Braz J Health. 2O14; 2(3): 151-156.

6. Gibson FA, Rezende SM, Braga GF, Cunha FM. Luxação traumática do joelho: análise clínica. Rev. méd. Minas Gerais. 2008; 18:93-9.

7. Ibrahim SA, Ahmad FH, Salah M, Al Misfer AR, Ghaffer SA, Khirat S. Surgical management of traumatic knee dislocation. Arthroscopy. 2008; 24(2):178-87.

8. Kupczik F, Schiavon MEG, Vieira LA, Tenius DP, Fávaro RC. Luxação do joelho: estudo descritivo das lesões. Rev. bras. ortop. 2013;48(2):145-151.

9. Kennedy JC. Complete dislocation of the knee joint. J Bone Joint Surg Am. 1963; 45:889-904.

10. Schenck R. The dislocated knee. Instr Course Lect. 1994; 43:127-36.

11. Schatzker J, McBroom R, Bruce D. Tibial plateau fracture. Clin Orthop.1979;138:94-104.

12. Berg K, Wood-Dauphinée S, Williams JI, Gayto D. Measuring balance in the elderly: preliminary development of an instrument. Physiotherapy Canada. 1989;41:304-11.

13. Tegner Y, Lysholm J. Rating systems in the evaluation of knee ligament injuries. Clin Orthop Relat Res. 1985;198:43-9.

14. Chhabra A, Cha PS, Rihn J et al. Surgical management of knee dislocations: surgical technique. J Bone Joint Surg Am. 2005; 87 (Suppl.1, Pt1):1-21.

15. Liow R, McNicholas M, Keating J, Nutton R. Ligament repair and reconstruction in traumatic dislocation of the knee. J Bone Joint Surg Br. 2003; 85(6):845-51.

16. Wong C, Tan J, Chang H, Khin L, Low C. Knee dislocations: a retrospective study comparing operative versus closed immobilization treatment outcomes. Knee Surg Sports Traumatol Arthrosc. 2004; 12(6):540-4.

17. Richter M,e cols. Comparison of surgical repair or reconstruction of the cruciate ligaments versus nonsurgical treatment in atients with traumatic knee dislocation. Am J Sports Med. 2002; 3O(5):718-27. 
18. Frobell RB, Roos EM, Roos HP, Ranstam J, Lohmander LS. A randomized trial of treatment for acute anterior cruciate ligament tears. $\mathrm{N}$ Engl J Med. 2010;363(4):331-42.

19. Almeida GPL, Gilvan OA, Amélia Pasqual Marques. Fisioterapia no tratamento conservador da ruptura do ligamento cruzado anterior seguida por ruptura contralateral: estudo de caso. Fisioter. pesqui. 2014:21(2):186-192.

2O. Rêgo AS, Salgado FHS, Santos HP, Aragão MB, Pereira SK. Atuação da fisioterapia no pré e pós-operatório de reconstrução do ligamento cruzado anterior (LCA). Revista Brasileira de Prescrição e Fisiologia do Exercício. 2014; 8(46): 367. 